\title{
INTEROPERABILITY AND STANDARDISATION IN COMMUNITY TELECARE: A REVIEW
}

\author{
R. Sethi*, D. Azzi*, R. Khusainov* \\ *Dept. of Electronic and Computer Eng., University of Portsmouth, UK. Correspondence email: richie.sethi@port.ac.uk
}

Keywords: telecare, standard, interoperability, framework, IEEE11073

\begin{abstract}
Interoperability and standardisation issues in community telecare differ from those pertaining to telemedicine and are examined with reference to older persons care. Issues discussed include devices, network transition, data characterisation, communication, data exchange, presentation, standards, protocols and certification (including IEEE 1107, ZigBee health Care, paSOS and the Continua Alliance). Standard requirements are reviewed and recommendations made, and FuTuRE, an open source telecare framework, is proposed.
\end{abstract}

\section{Introduction}

Telemedicine (clinical care by means of live teleconsultation or monitoring of clinically-relevant health parameters) can be distinguished from community telecare (social care, including emergency alarms, dementia care, assisted living, and longer term wellbeing management). The commonality between the two is in equipment and telecommunications, thus they share some standards and interoperability issues such as in medical equipment and wireless technologies.

Community telecare however differs significantly in its service architecture and provision and can involve highly (sensory) data-intensive applications. The growing demand for low cost IP-based telecare services, resulting from an increasing elderly population and strains on community care services is affected by a lack of standards and problems with interoperability.

There are presently no open communication standards specifically for telecare and most commercial remote monitoring protocols are proprietary [1] and whilst there have been efforts at producing unifying health monitoring profiles, the operation paradigm in telecare is usually very different [2], requiring significant application development.

Interoperability issues have resulted from lack of common standards and proliferation of proprietary wireless technologies such as ZigBee and other mesh and sensor network protocols. Commercial wireless sensors are rarely compatible between manufacturers, don't work outside of dedicated Personal Area Networks and do not transition seamlessly between fixed and mobile networks. There are also problems with Human Factors. The lack of specific (and open) standards also makes it difficult and expensive for manufacturers to bring newer IP-based telecare systems to market, instead developing non-interoperable proprietary solutions. This lack of standardisation and thus interoperability has prevented consumer-driven mass production and investment in newer technologies, which means that care needs could be better addressed, although efforts have been made to harmonise standards for independent living applications and for medical information technology in general.

This paper discusses the issues involved in interoperability and standardisation specific to community telecare for elderly users (rather than clinical telemedicine), and makes recommendations. 'Telecare' identifies the technical issues involved as a result of the lack of a unifying standard, including issues with devices, network transition, data characterisation, communication and data exchange and presentation. 'Standards, Protocols and Certification' looks at attempts at producing unifying standards including IEEE 1107, ZigBee health Care, paSOS and the Continua Alliance. Finally, interoperability and standardisation requirements are reviewed and recommendations made for a future standard and an open source telecare framework is proposed.

\section{Telecare}

Most community telecare systems rely on ambulatory monitoring using mobile and fixed sensors to send measurements to a monitoring centre, supporting social care packages and preventing hospital admissions by early intervention. The process consists of periodically acquiring either biomedical signals [2] (e.g. heart rate) or event data (e.g. falls or enuresis) for real-time processing and detection of abnormal behaviour, which in turn triggers an alarm, allowing corrective action to be taken by a carer.

Pendants and fall detectors are the most common devices found in this setting and are typically worn on the body, with it being increasingly common for sensors to be incorporated into clothing, bracelets' [2] etc., but there is also a wide range of safety and security devices to detect parameters such as smoke, ambient temperature and location which are usually fixed in a user's home. The regions in the immediate vicinity of a user and that within range of a low-power transmitter are termed as a Body Area Network (BAN) and Personal Area Network (PAN) respectively. There are three essential elements in an IP-based telecare system (see figure 1):

1. The end-monitoring devices located in the BAN or PAN which collect sensory information, 
2. The conduit for data exchange such as a fixed internet gateway or mobile cellular device which exists in a Local or Wide Area Network (LAN/WAN) and

3. The remote processing server, where alarms are raised, voice and video decoded and event data integrated into care package records.

The key difficulty in this scenario is in homogenous integration [2] between the three areas. For homogenous operation, devices based on any technology should be able to a) integrate into existing BAN and PAN networks with mobile devices seamlessly transitioning between these and LAN and WAN networks, b) transmit data in a recognised format to the remote server and process and c) store and present this data in a predetermined and user friendly format for external data exchange (such as for health records and for sharing data with third party applications or organisations) or for presenting data via a user interface.

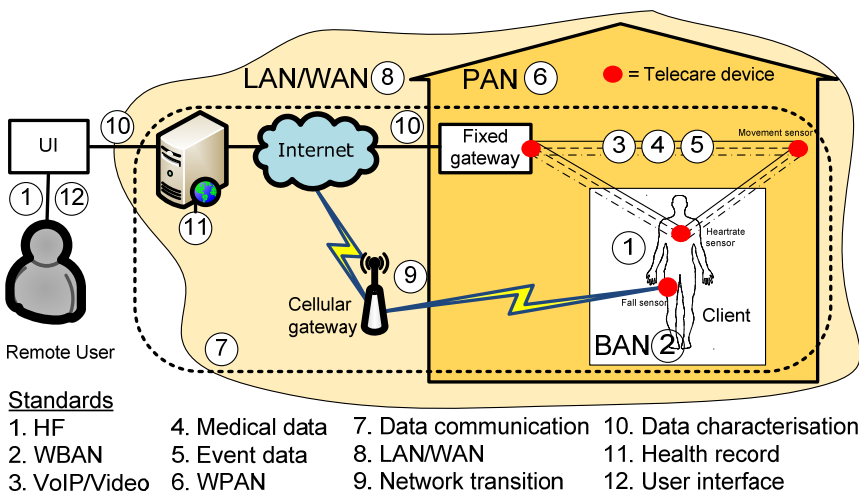

Figure 1: Standardisation in Telecare

Figure 1 lists 12 standard areas which could come into operation in a typical IP-based telecare system. The core issue is that data communication involves interaction at different OSI layers [3]; with layers 1-4 being managed by the underlying standards of the communication technology and layers 5-7 by the software API and user application, possibly defined by HL7 standards [3]. There is therefore a need for an end-to-end specification of how devices interact with the rest of the telecare system and transport and present information in a standard format for use, both for use in an end application and for external transfer. There are many individual standards involved in health information systems, but none specifically for telecare [2].

\subsection{Devices and Network Transition}

There are a wide range of low power wireless technologies, such as ZigBee, Bluetooth and Low-Power Wi-Fi, which are built on top of international standards for layer 1-4 communication, and others which use entirely proprietary technologies. Whilst individual wireless consortiums ensure interoperability between certified devices, interoperability between device manufacturers is yet to be resolved. There are two issues involved with homogenous operation: First, that there needs to be a common way to identify devices and share information, regardless of the underlying technology used and second, data exchange protocols or APIs which enable multiple transport technologies to exchange data. These two have been largely resolved by ISO/IEEE 11073 Personal Health Data (PHD) Standards, the ZigBee health profile and the Continua Alliance, which are discussed in this paper.

Different wireless technologies also use different RF spectrum ranges. These may be defined by the underlying communication standards. IEEE 802.15.4 operates in the ISM band; $868 \mathrm{MHz} @ 20 \mathrm{kbps}$ (Europe) and 2.4GHz @ $250 \mathrm{kbps}$ (Global). IEEE 802.11n also operates in the global ISM band, with a maximum data rate of $288.8-600 \mathrm{Mbps}$. Although the spectrum for IEEE 802.15.6 (BAN) is not presently available, it is expected to operate in the ISM band [4]. The benefit of using a higher frequency is twofold: greater bandwidth and a wider spectrum of operation. The BS EN 50134 standard for social alarms, which all telecare manufacturers comply with, however, specifies the use of dedicated social alarm frequencies (869.20-869.25 MHz in the EU) for the purposes of exclusive operation. The problem is that these bands were chosen at a time when requirements were low data rate and bandwidth. No mainstream wireless standards operate in these bands in the EU, which means that most telecare products have to use proprietary RF protocols, thus losing out on the benefits of the 11073, ZigBee and Continua efforts. As manufacturers begin to adopt ISM-based technologies for next generation IP-based products, another issue arises out of interference with a wide range of consumer electronic products which use this band, with reports of interference between 802.15.4 and 802.11 technologies [5]. It is also noteworthy that existing spectrum allocation for social alarms has been withdrawn [6] and will be phased out.

Second, telecare is typically designed to work in a fixed geographical area, commonly a residence, the range of which is limited by the PAN, or in some cases LAN, where multiple PANs interact. However, older persons on average spend $25 \%$ of their waking hours up to $60 \mathrm{~km}$ away from their homes [7] making 1030 journeys a year [8] and a third to half of falls occur outside the home [9] [10], which stresses the need for mobile telecare devices. Such devices usually encode data using a proprietary format and transmit this via SMS (GSM) or GPRS/3G (for higher volumes of data) and usually include GPS coordinates for location. The paSOS protocol aims to resolve mobile data communication and is discussed further in this paper. In heterogeneous systems, devices should seamlessly transition between the fixed geographical location (BAN/PAN) and mobile networks (LAN/WAN), however this is made difficult by a lack of telecommunication standards to allow for seamless transition between network architectures. The success of hotspots in cellular networks suggests that seamless operation may be extended to different network architectures. Matoba and Kim [11] suggest a method to deploy a WLAN with mesh topology for Internet access using internal L3 routing with NAT, which resolves the issue of regular L3 routing, wherein all network nodes need a public IP address, requiring routing tables of internet routers to be updated; which is not feasible when using a generic hotspot. Such a system must also be capable of using the same IP stack and resolving routing and addressing issues 
in situ, however the need for a different IP stack and support for additional protocols may increase the footprint of the device firmware.

\subsection{Data Characterisation and Communication}

Data characterisation protocols encode data for transmission to remote interconnecting systems. Telecare involves three types of data; event information, medical data and device information along with VoIP or Video traffic. The use of proprietary signalling protocols by telecare manufacturers impedes interoperability and the mix-and-match of products. Furthermore, the upgrade of technologies or switching of manufacturers may involve cost-prohibitive changes for local authorities or service suppliers, in terms of application software or alarm receiving hardware. Additionally, equipment tests have shown that a significant proportion of systems using proprietary signalling protocols do not operate reliably on next generation IP-networks [12]. The paSOS protocol specifies a data format for mobile systems.

There are also concerns with Quality of Service (QoS), especially in consumer broadband circuits with shared bandwidth. QoS is important in telecare systems for the reliable transmission of signals in emergency situations [13]. In the entire data communication system, it is difficult maintain QoS across the different communication technologies as data passes through OSI layers on several occasions and QoS implementation varies. Bui et al. [13] propose providing real-time QoS for peer-to-peer multi hop communication in 802.15.4-based systems, at the MAC layer.

\subsection{Data Exchange and Presentation}

Alarm receiving centres or remote data management servers, possibly employing data mining techniques, process sensory and event information. There is need for the data format to be interoperable with external systems such as e-health patient records, to support the management and delivery of health services. Health informatics is generally interpreted as being in the Application OSI layer [3] and HL7 standards such as the Clinical Document Architecture (CDA) provide an exchange model for health information, although the clinicaloriented nature of the architecture hinders it's effective use with social care service management; record management of client-carer interaction to support the delivery of social care services often store data other than clinical information.

Many service users including older people and those with reduced cognitive function often find it difficult to operate newer technology and standard methods of presenting data using Human Factors (HF) may be used in the design of the user interface, to ensure both ease of use as well as providing a standard format for social carers to interact with, improving consistency and further reducing training costs and possibly the likelihood of errors, due to missing out vital information when moving between systems. The ETSI publishes HF recommendations for telecare (ETSI TR 102 415:2008).

\section{Standards, Protocols and Certification}

\subsection{ISO/IEEE 11073 Personal Health Data (PHD) Standards}

The 11073 PHD group of standards cover several aspects of defining medical data from device to server and defines a common framework for making personal health data available in 'transport-independent transfer syntax' [14], which includes device information and status, nomenclature, device specialisations and a communication protocol (see figure 2). The core functionality is at layer 7 and abstraction between communication and Application layers is provided by an Optimised exchange protocol (OEP) which allows interaction with multiple communication technologies. It supports Application layer services at one end for connection management and reliable transfer of data as well as data exchange, at the other, which defines commands, configuration information, data format and overall protocol management [14].

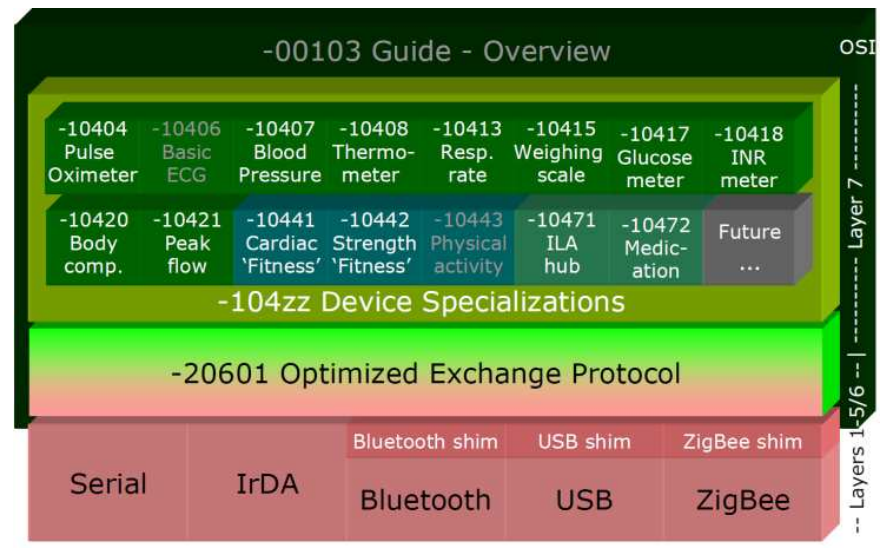

Image 2: ISO/IEEE 11073 framework [3]

Device types are given a unique classification and data is formatted according to device type. The Independent Living Hub (ILH) device specialisation (11073:10471) relates specifically to telecare equipment, including emergency button and fall, motion and environment sensors.

\subsection{ZigBee Health Care}

The ZigBee Health Care Application Profile uses the 11073 PHD communication protocol and device specialisations, with the Transport layer being supplied by ZigBee, which includes device discovery, security, location and voice services. The 11073 ILH device specialisation allows for telecare equipment to be classified and data format standardised. Furthermore, physical environments are also classified ranging from a 'courtyard' to 'fountain', along with object values such as a 'toilet' and 'microwave' [15]. This enables encoded data to be exchanged with interoperable systems. Furthermore ZigBee supports VoIP as part of its 'Ageing independently device profile' [15]. It further supports the pairing of devices at the Application layer, with an optional security cluster- either Alpha-Secure Access Control (ASAC) or Alpha-Secure Key Establishment (ASKE). 


\section{3. paSOS}

The paSOS protocol, produced by a consortium of telecare and telecommunications organisations, aims to provide a standard data protocol for mobile telecare services. This provides for a data format (see figure 3) to standardise data exchange over mobile networks between a mobile device and the remote service. Emergency alarms are defined by an 'alarm frame' [16] which encodes data to be transmitted via SMS and includes location information, which can further be updated via a position request. The protocol also handles frame identification, acknowledgement and error handling.

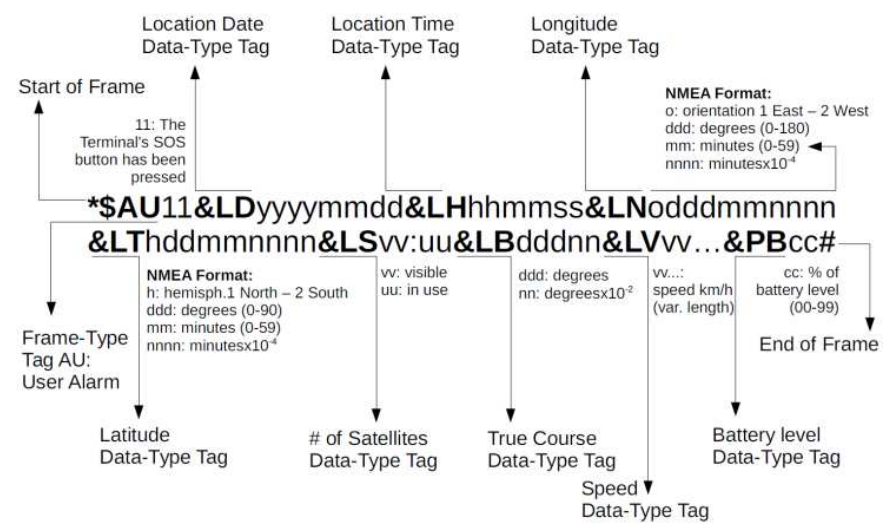

Figure 3: paSOS Alarm Frame [16]

Version 1 of the protocol supports SMS as a bearer service, and the next revision, due for release in mid-2011 will also implement GPRS/UMTS, which is crucial for data intensive applications such as real-time tracking and sensor measurements. The protocol does not, however, support devices capable of functioning in both fixed and mobile networks and transitioning between them.

\subsection{Continua Alliance}

The Continua Health Alliance is a non-profit alliance of healthcare and technology companies which selects technologies and standards and publishes guidelines to promote interoperability, which is guaranteed through its certification process [17]. Bluetooth, ZigBee, USB, IEEE 11073 and HL7 standards are supported. It uses a selection of standards not specifically developed for community telecare, but whilst the system is geared towards telemedicine overall, 11073 and ZigBee support telecare applications. Specified technologies must be used, and it follows that the core focus is on interoperability rather than the most technically optimal or feature-rich solution.

Furthermore, an end-to-end solution is not provided and it is still up to the manufacturer to implement the software solution, although application examples are provided, which can reduce development cost. Additionally, it does not resolve technical issues such as the lack of an IP-based social alarm standard and dedicated RF spectrum.

\section{Requirements and Recommendations}

\subsection{Interoperability and Standardisation Requirements}

Interoperability can be defined as functional (Shared architectures, methods and frameworks) and semantic (Shared data types, terminology and coding) [3] and standards promote interoperability, along with providing value addition, regulation, safety and a cost saving [18]. Although standards organisations and industry consortia are increasingly promoting standards-based interoperability in remote health applications, it may be said that their focus is too generic and don't resolve issues involved specifically with community telecare applications, and that this hinders the implementation of telecare services supported by the ZigBee Health Care application profile and IEEE 11073 PHD standard.

There is a need for industry collaboration to agree on a common telecare framework to support the development of next generation IP-based services. Requirements include interoperability across all 7 OSI layers, from sensor to monitoring application to support end-to-end system management, the implementation of QoS to support critical data transmission over shared broadband connections, a telecommunications protocol for seamless network transition of fixed-mobile devices and Real-Time Location Tracking Systems (RTLS), which are partly solved by HL7 standards [3], but only at the Application layer. Furthermore, the European Communications Office must update the harmonised social alarm RF spectrum to support next generation IP-based services.

The development of a standard typically involves compromise from parties involved in the working groups and thus may not be the most technically robust solution, however the BSI can fast-track sponsored standards [19]. The typical standards-development process is shown in figure 4.

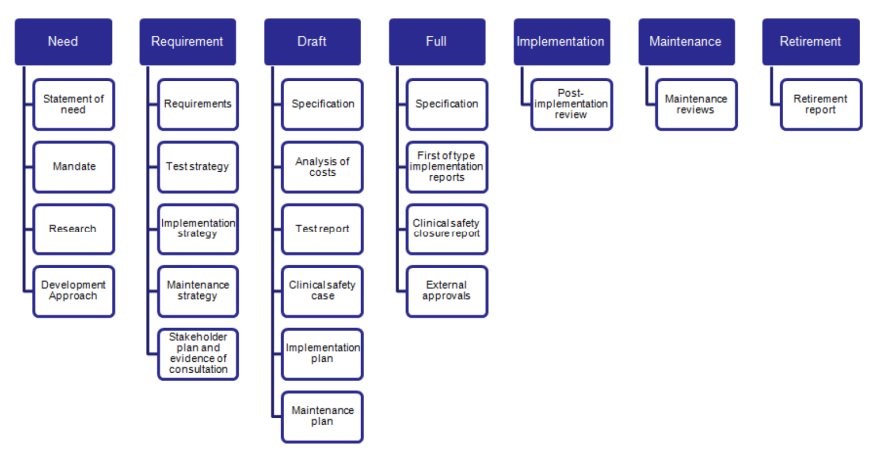

Figure 4: Standards Development Process [18]

Severs [18] argues that a good standard must be useful, implementable, interoperable, compatible and able to anticipate the cost burden and promote organisation safety. A five-point recommendation for a good telecare standard is proposed as follows:

1. Interoperable- support for mainstream as well as proprietary communication technologies

2. Flexible- modular form which allows manufacturers to choose from a range of functionality 
3. Open source- dynamic community development and collaboration

4. Low investment- minimal need for systems development in the integration with existing systems

5. Future proof- modular form which can be easily revised, considering future technologies and formats

\subsection{Open Source Telecare Framework}

FuTuRE (Framework for TelecaRE) is an open source framework specifically designed for community telecare, presently under development at the University of Portsmouth, which allows for end-to-end homogenous integration with seamless transition between network architectures. Such a framework will reduce development time and therefore timeto-market and therefore allow for rapid commercial development and deployment in the older person telecare industry and will support multiple communication technologies. In modular form for flexibility, manufacturers may chose which features to implement and enabling open source community collaboration (rather than closed memberonly consortium development), reduces development cost and consumer prices, encourages competition, enhances the effectiveness of applications and thus provides better quality solutions to end users.

Consisting of a decentralised 'triage' model, where task agents assign event priorities, with data frames encoded as Tagged Priority Information Frames (TPIF), the framework should result in greater reliability, a reduction in communication overheads and increased AI-efficacy [20]. QoS is implemented to improve the reliability of critical data transfer, and VoIP, video and IPv6 are supported to ensure future-proof operation. The framework will additionally support devices capable of functioning in both, fixed and mobile networks and define a protocol for transitioning between them.

Research identifies the existence of 'middleware' that 'abstracts network or hardware specific tasks to the application' [21] and which allows data to be sent to any node of the network without considering its location or sending broadcast messages, using a simple and unique interface (based on query-response), that can be dynamically initiated in any node at any time. Middleware abstracts hardware to software and thus application development and service delivery. The middleware model proposed by Martinez et al. [22] for example, proposes a standards-based, plug-and-play platform for telemonitoring. Although the proposed model is entirely based on one standard, restricting the usefulness of their model, an attempt has been made at multi-platform support. It also focuses on medical data and devices for clinical-based 'telemedicine', as opposed to social care-based 'telecare' and is not generic, seamless and does not form a framework for rapid development and deployment.

However whilst several authors propose using middleware to enable the end-to-end management of services, by acting as a universal architecture [21] [23], instead of managing events, middleware only defines the basis for events e.g. how data can be translated from sensor to agent and is, on its own, not practical to implement in an industrial environment and may not offer enough flexibility to cater to varying product needs. Further methods are therefore required to extend the benefits of middleware to a practical framework.

Figure 5 illustrates the major components of the proposed framework, which coexists with existing standards and protocols and exists in a 'third dimension', using middleware to enable end-to-end management and further supported by protocols. An Application Data Exchange protocol extends HL7 standards to telecare systems, for interoperability with external systems and a Telecare Management protocol is responsible for core functionality.

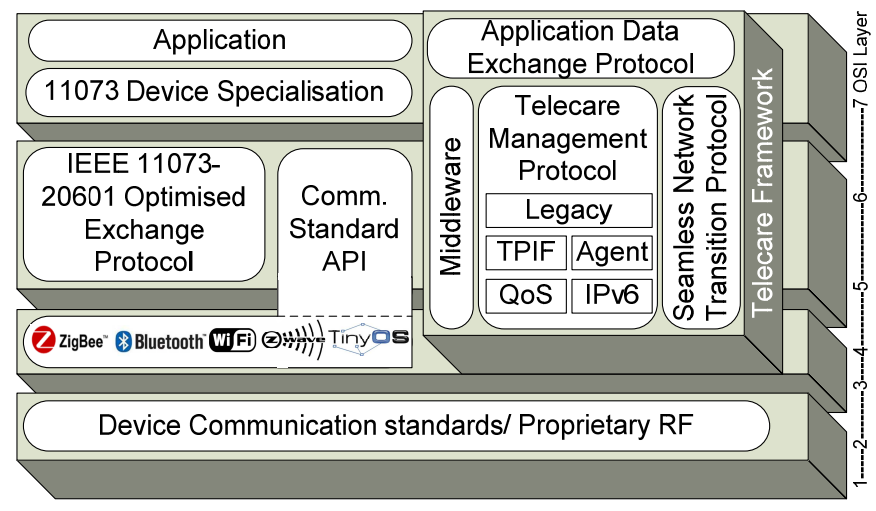

Figure 5: FuTuRE Telecare Framework

Tablado et al. [24] recognise the need for an 'anywhere, anytime' alarm trigger which can work outside of the home and that pre-processing can be done before wireless transmission, but do not solve the problem of seamless network transition. A protocol is provided as part of the framework to enable seamless network transition for homogenous telecare devices, based on the paSOS protocol for data characterisation and which will support GSM, GPRS and UMTS.

\section{Conclusion}

The development of next-generation telecare products are hindered by the lack of platform-independent standards and problems with interoperability. Although recent and ongoing standard and protocol developments and certifications schemes will assist in improving development, there are still unresolved problems. There is a case for a new unifying standard and the open source FuTuRE framework is proposed, which aims to resolve these issues.

\section{References}

1. Social Services Improvement Agency. Investigation of the potential for the joint procurement of telecare equipment and maintenance. 2007.

2. An Updated Review of the Applicability of ISO/IEEE 11073 Standards for Interoperability in Telemonitoring. Galarraga, M., et al. Lyon: IEEE, 2007. 29th Annual EMBS Conference. 
3. Reynolds, M. The Need for Interoperability \& Technology Standards in Telecare. : SEHTA, 2010.

4. Drude, S. IEEE P802.15 Working Group for Wireless Personal Area Networks (WPANs). 2006.

5. Minimizing 802.11 Interference on ZigBee Medical Sensors. Hou, et al. Los Angeles: s.n., 2009. Fourth International Conference on Body Area Networks.

6. Electronic Communications Committee. ECC/DEC/(08)02: ECC Decision on the withdrawal of ERC/DEC(97)06. 2008.

7. Elderly People and the City: An Investigation with GPS Methodology. Shoval, N. [ed.] H Harder, T Nielsen and J Majg. Copenhagen: Aalborg Universitet, June 24th 2009. Urbanism \& Urban Qualities. 1603-6204.

8. Quality of life for the elderly: the transport dimension. Banister, D and Bowling, A. 2, s.1. : Elsevier, April 2004, Transport Policy, Vol. 11, pp. 105-115. 0967-070X.

9. Day, L, Kent, S and Fildes, B. Injuries among older people. Hazard. Edition No. 19, 19 June 1994, Vol. 1, pp. 116.

10. Lewis, P. Preventing falls in older people. Injury Issues. 5 February 1992, pp. 1-3.

11. Interconnecting Multi-Vendor Multi-Hop WLAN and Internet via Hotspot. Matoba, A. and Kim, M.W. s.l. : IEEE, 2009. ICACT .

12. Ofcom. Next Generation Networks. 2009.

13. Bui, B., et al. Adaptive and robust QoS in wirelessenabled sensing devices for Assisted Living. s.1. : University of Illinois at Urbana Champaign, 2006.

14. ISO/IEEE. 11073-20601: Application Profile-Optimised Exchange Protocol. s.1. : IEEE, 2008.

15. ZigBee Alliance. ZigBee Health Care Profile Specification VIR15. 2010.

16. TECSOS. paSOS protocol description version 1.0. 2010.

17. Continua Health Alliance. The Next Generation of Personal Telehealth is Here. 2010.

18. Reynolds, M. The contribution of health information standards to telecare. s.1. : Information Standards Board for Health and Social Care.

19. Alcock, S. The potential of British Standards in telecare. s.1. : SEHTA, 2010.

20. Community Telecare Technology. Sethi, R., et al. Portsmouth: The University of Portsmouth, 2010. Health Development Forum Showcase.

21. PERSEIA: a Biomedical Wireless Sensor Network to Support Healthcare Delivery for the Elderly and Chronically Ill. Jimenez-Fernandez, S., et al. Engineering in Medicine and Biology Society, 2006. EMBS '06. 28th Annual International Conference of the IEEE. Vols. Aug. 30-Sept. 3 2006, pp. 2064-2066. 10.1109/IEMBS.2006.259247.
22. Standard-Based Middleware Platform for Medical Sensor Networks and u-Health. Martinez, I., et al. 3-7 Aug. 2008. Computer Communications and Networks, 2008. ICCCN '08. Proceedings of 17th International Conference on. pp. 1-6. 10.1109/ICCCN.2008.ECP.135.

23. Middlecare: middleware technology for home-based chronic patient shared care. Gomez, E.J., et al. s.l. : IEEE EMBS, 23-26 Oct. 2002. Engineering in Medicine and Biology, 2002. 24th Annual Conference and the Annual Fall Meeting of the Biomedical Engineering Society, EMBS/BMES Conference, 2002. Proceedings of the Second Joint. Vol. 3 , pp. 1900- 1903. 10.1109/IEMBS.2002.1053084.

24. Intelligent monitoring of elderly people. Tablado, A.; Illarramendi, A.; Bermudez, J.; Goni, A.;. s.l. : IEEE EMBS, 2003. Information Technology Applications in Biomedicine, 4th International IEEE EMBS Special Topic Conference on. Vols. 24-26 April 2003, pp. 78- 81. 10.1109/ITAB.2003.1222447. 